\title{
Redes profesionales e interdisciplinariedad en trabajo social comunitario: una respuesta integral a problemas sociales multidimensionales
}

\author{
María Dolores Rodríguez Álvarez \\ Ayuntamiento de Getafe (Madrid) \\ <lola-rodriguez@hotmail.es>
}

\begin{abstract}
Autonomia erkidegoetako administrazioek zein tokikoek kudeatutako gizarte-zerbitzu komunitarioak ongizate-estatuaren tresna bat dira, eta gizartelanaren alorreko profesional ugarik jarduten dute zerbitzu horietan; eta hainbat administrazio eta zerbitzuetako profesionalekin koordinazioan egiten dute, gainera, beren eguneroko lana. Horrek ondorioz dakar, beraz, zeharkako lana eta sarean lan egin beharra. Artikulu honetan gizarte-lan komunitarioari buruzko ikerketa baten emaitzak aurkezten dira, zehazki diziplinarteko lanaren definizioa eta gizarte-langileei eta Madrilgo Autonomia Erkidegoko hainbat udaletan beren zerbitzuak ematen dituzten beste profesional batzuei egindako elkarrizketa erdiegituratuen emaitzak. Bertan bildutako datuetatik ondoriozta daiteke diziplinartekotasunak konfiantzazko etika bat dakarrela ondorioz, giza harremanen printzipioan eta esku-hartze profesionala agintzen duten balioak erdibanatzean oinarritua, eta orobat dakarrela lanaren emaitzak aberastea.
\end{abstract}

\section{GAKO-HITZAK:}

Gizarte-lan komunitarioa, diziplinarteko lana, tokieremua, sare-lana.
Los servicios sociales comunitarios gestionados por las administraciones autonómica y local son un instrumento del Estado de bienestar con una importante presencia de profesionales del trabajo social, cuya práctica profesional cotidiana se realiza en coordinación con profesionales de distintas administraciones y servicios, lo que implica realizar un trabajo transversal y en red. Este artículo presenta los resultados de una investigación sobre trabajo social comunitario que pretende conocer la definición del trabajo interdisciplinar y los niveles de intervención a partir de los resultados de entrevistas semiestructuradas realizadas a trabajadores sociales y a otros profesionales que prestan sus servicios en el ámbito local en diferentes municipios de la Comunidad de Madrid. De los datos recogidos cabe inferir que la interdisciplinariedad supone una ética de confianza, basada en el principio de la relación humana y en compartir los valores que rigen la intervención profesional, y supone un enriquecimiento de los resultados del trabajo.

\section{Palabras ClaVe:}

Trabajo social comunitario, trabajo interdisciplinar, ámbito local, trabajo en red. 


\section{Introducción}

Los servicios sociales gestionados por las administraciones autonómica y local pueden considerarse un instrumento del Estado de bienestar destinado a la satisfacción de necesidades ciudadanas con el fin de aumentar el bienestar social. Para su efectividad, requieren una protección suficiente y “deben posibilitar, no sólo la subsistencia, sino también procesos de desarrollo personal" (Casado, 2007: 280). Siguiendo a Gutiérrez Resa y Uña (2010: 16-17), "hablamos de un sistema público de servicios sociales como conjunto integrado y coordinado de programas, recursos, actividades y equipamientos destinados a la atención social de la población y gestionados por la Administración autonómica y local". Las leyes de servicios sociales de las comunidades autónomas se han ocupado de las competencias municipales, e igualmente el Plan Concertado de 1988 entre las tres administraciones (central, autonómica y local) ha desarrollado una red de servicios sociales en toda España, fruto del acuerdo entre la Administración Central, las comunidades autónomas y la Federación Española de Municipios y Provincias. Los avances del Plan Concertado se pueden determinar por su implantación en el territorio, lo que en una red básica de servicios sociales viene a significar, para Subirats (2007), la garantía de proximidad a la población y un acceso a los recursos que ha alcanzado el $98 \%$ de la población, como promedio, y el $100 \%$ en la mayoría de las comunidades y ciudades autónomas. La ventaja de las políticas de proximidad en los servicios sociales reside en que permiten 'ajustar' el sistema a las necesidades de su entorno, detectar estas necesidades y también identificar y dinamizar los recursos de la propia comunidad: "así sucede en gran parte de los países europeos, sin haber ocurrido en España por la fuerte tradición centralizadora y por la escasez de recursos económicos de la Administración local” (Alemán, 2010: 204). La Ley 11/2003, de 27 de marzo, de Servicios Sociales de la Comunidad de Madrid recoge, en su artículo 2, la finalidad de tales servicios:

1. Los servicios sociales tendrán por finalidad la promoción del bienestar de las personas, la prevención de situaciones de riesgo y la compensación de déficits de apoyo social, centrando su interés en los factores de vulnerabilidad o dependencia que, por causas naturales o sobrevenidas, se puedan producir en cada etapa de la vida y traducirse en problemas personales.

2. El objetivo de los servicios sociales es el de asegurar el derecho de las personas a vivir dignamente durante todas las etapas de su vida, teniendo cubiertas las necesidades sociales.

3. A los efectos de lo regulado en esta Ley, se entienden como necesidades sociales las derivadas del derecho de la persona a realizarse como ser social en el ámbito convivencial, interpersonal y familiar, y en el relacional, entre el individuo y su entorno social.

En el artículo 24, punto 2, relativo al modelo de intervención, establece que la “intervención en servicios sociales tendrá carácter interdisciplinar al objeto de ofrecer una atención integrada. El número y composición concreta de los distintos equipos interprofesionales de los que podrán formar parte, entre otros, trabajadores sociales, psicólogos, sociólogos y educadores sociales, se establecerá en función de los objetivos y la naturaleza de cada centro o servicio". Finalmente, en el apartado de las funciones que corresponde desarrollar al nivel de la atención social primaria (artículo 31, j), señala, entre otras, la “coordinación con el nivel de Atención Social Especializada, así como con otros servicios para el bienestar que operen en el mismo territorio, de manera especial con los de salud, educación, cultura y empleo, con el fin de favorecer la atención integral de las personas".

Por otra parte, el contexto en que se desarrolla la acción social local "constituye una dimensión intrínseca e histórica del trabajo social y del trabajo con las comunidades, que dio origen a un método específico de intervención: el trabajo social comunitario" (Cardoso, 2012: 107), y éste toma, a su vez, diferentes significados e interpretaciones como uno de los tres métodos de intervención clásicos, junto al individual y grupal, o más recientemente, como un proceso dialógico dentro del continuum metodológico individuo-grupo-comunidad. Es importante destacar que el trabajo comunitario no debería ser un programa ajeno al resto, sino "una herramienta de intervención transversal a todos los programas, complementaria del resto de intervenciones del nivel individual o familiar, y servir a sus objetivos de manera articulada" (Rodríguez Cabrero, 2011: 267). No podemos dejar de señalar que, en nuestra sociedad contemporánea, los problemas "se caracterizan por una diversidad de dimensiones que interactúan, junto a las personas, las organizaciones y las comunidades locales, de manera trasversal e interdisciplinar sobre las cuestiones sociales y los saberes disciplinares" (Ferreira, 2009: 58), lo que hace necesario aprender a trabajar en red y de forma interdisciplinar para dar una respuesta integral a los problemas sociales actuales, que tienen carácter multidimensional. De la Red (1997: 544) afirma que, desde el trabajo social, se verifica con frecuencia el carácter complejo de las necesidades, y ello le plantea nuevas exigencias, como "el paso de una visión sectorial y particular de los problemas en los que los servicios sociales intervienen, a una visión interdisciplinar de los mismos". De Robertis (2006: 315) considera que los problemas sociales, lejos de disminuir, se agravan ante la deconstrucción de las políticas sociales y la multiplicación del desempleo y el aumento de la precariedad: "la capacidad de creación y de llevar adelante nuevas iniciativas muestra la vitalidad de la profesión, su compromiso en la lucha por la justicia social y con la democracia”. Igualmente, es necesario destacar que "la elevada fragmentación de políticas, programas y proyectos en distintas administraciones ha hecho que la transversalidad y el trabajo en red se impongan como necesidad" (Subirats, 2007: 81).

La acción del trabajador social en las redes sociales de nivel local asume tres dimensiones, porque se dirige 
al mismo tiempo a la persona, a la organización de los servicios y al territorio (Ferrario, 2009: 70):

- Con la persona, el trabajador social trabaja con redes; integra, forma redes; forma o favorece y sostiene grupos de autoayuda.

- En la organización de los servicios, favorece las relaciones institucionales, crea una praxis de colaboración en lo cotidiano en torno a los proyectos, diseña proyectos de red.

- En el territorio, genera encuentros en lo cotidiano; construye conexiones en el marco de los proyectos, entre recursos y con el servicio; estimula su potenciación.

En el territorio, desde una perspectiva ecológica, se desarrollan nexos relacionales entre personas, $y$ entre éstas y su entorno, y desde este punto de vista, "el concepto de comunidad se refuerza con la idea de territorialidad, de territorio como lugar de acción abierto, de par en par, al milagro de lo relacional, de lo comunitario emergiendo" (Navarro, 2004: 312), porque el trabajo en redes está formado por el conjunto de las intervenciones de conexión de recursos y de estrategias cuyo objetivo es producir nexos en las relaciones significativas y procesos de crecimiento que se activan dentro de esos mismos recursos, para conseguir mejorar el nivel de bienestar de las personas y de los colectivos.

La perspectiva de red aplicada a los servicios (Ferrario, 2009) concierne a diferentes campos: la conexión de los servicios que forman parte de ella, la conexión de diferentes sectores -sociales, educativos- en una misma área territorial y la conexión entre profesiones o entre los miembros de una profesión. La Figura 1 muestra los actores a los que se dirige la intervención del trabajador social en el ámbito local (individuo, familia, comunidad), entre quienes existen vínculos, lazos o relaciones y se puede contar con un número de actores diferente, entre quienes existen diferentes tipos de relaciones. El trabajador social tiene la tarea prioritaria de activar los recursos de las personas (capacidades) y de los diferentes contextos, ya sea ambientales (redes de apoyo social) o sociales (servicios, programas y prestaciones) desde las tres dimensiones señaladas: el desarrollo personal (la relación de ayuda), el desarrollo social (la solidaridad) y el desarrollo organizacional (políticas públicas). La red social se convierte así en una perspectiva que nos ofrece claves interpretativas de la complejidad social y estrategias de intervención para afrontarlas, y su valor es la capacidad de generar cambios y mejoras sociales, $y$, por tanto, convertirse en un elemento de construcción de la ciudadanía social.

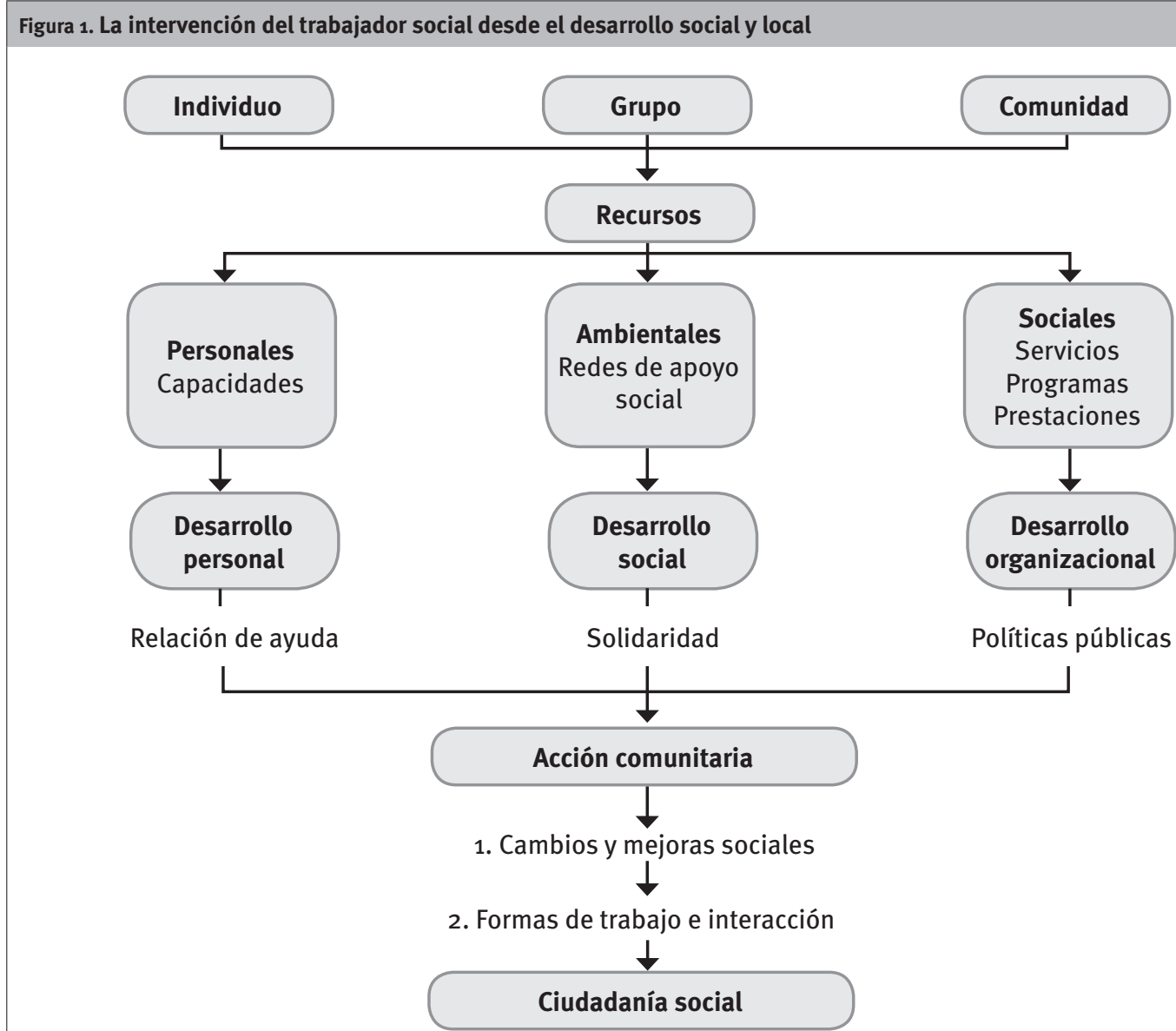

Fuente: Rodríguez Álvarez (2014). 
No podemos dejar de señalar que el trabajo en redes pone en relación, de forma circular y abierta, los recursos naturales de las personas, familias, grupos y organizaciones comunitarias, y a éstos, a su vez, con los servicios y programas públicos, privados y concertados que se ofrecen a través de los profesionales. Presentamos seguidamente algunas consideraciones en torno al trabajo interdisciplinar que consideramos relevantes en el marco de nuestra investigación:

- Produce cambios en el proceso de toma de decisiones: el trabajo desde las redes sociales impulsa nuevas formas de comprender la sociedad y supone una nueva práctica profesional, en la medida en que "ha cambiado su papel como responsable en la gestión de los problemas de las personas y las familias por el de coordinador y gestor de los recursos sociales de apoyo y ayuda a la persona y a la familia con necesidades, dejándoles la responsabilidad individual de su problema" (Ferreira, 2011: 235). La palabra inglesa para 'equipo', team, viene del inglés medieval, donde tenía el significado de 'familia' (Rygaard, 2008). Construir un equipo significa "construir un sistema de relaciones interpersonales suficientemente sólidas como para crear un sentimiento de identidad profesional común y también como para poder concentrarse en una tarea y trabajar a pesar de las diferencias de actitudes, de roles y de autoridad en el grupo" (ibídem: 283).

- Hace necesario compartir conocimientos y liderazgo: los problemas sociales son multidimensionales, lo que implica la necesidad de contar con equipos de trabajo formados por profesionales especializados para la intervención directa y para la planificación, diseño y evaluación de los programas sociales. Así, junto a los trabajadores sociales hoy trabajan profesionales de otras disciplinas, dependiendo de la especificidad de cada programa o servicio. Como indican Llena, Parcerisa y Úcar (2009: 93), “únicamente desde la interacción entre los diferentes profesionales y mediante el diálogo de los diversos saberes disciplinarios -educación, psicología, sociología, antropología, pedagogía y trabajo social-, resultará posible dar respuestas que sean a un tiempo integrales, apropiadas y ajustadas a las realidad de nuestro tiempo".

- Exige reconocer la capacidad de todos los agentes implicados: la intervención en redes nos plantea el paso de una metodología de la intervención multidisciplinar a una metodología interdisciplinar, considerada "un encuentro entre diferentes disciplinas y diferentes realidades con el objetivo de producir cambios en la metodología de la intervención (pasar de la multidisciplinariedad a la interdisciplinariedad)" (Ferreira, 2011: 284). En la intervención en redes, el profesional se ve como sujeto inserto en las relaciones sociales para fortalecer las relaciones de dichos sujetos, ya sea para la ampliación de su poder, su saber o de sus capitales. "Se trata", dice Faleiros (2003: 24), "de una teoría relacional del poder, de una teoría relacional de construcción de la trayectoria".

- Es un proceso de co-construcción (labor conjunta de todos los implicados en el problema/solución): cuando observamos la atención primaria y, en general, los servicios sociales (Llobet, 2004), nos damos cuenta de las enormes dificultades que supone generar un trabajo en red desde una estructura y una cultura organizativas no pensada para funcionar en red. La intervención en redes sociales supone un cambio de paradigma teórico que conlleva una labor conjunta entre los profesionales y genera un cambio de roles, la horizontalidad en la acción, cambios en el proceso de toma de decisiones, compartir conocimientos y liderazgo: en suma, reconocer las capacidades de todos los demás agentes implicados. Consideramos fundamental otra implicación, que Vega (1997: 197) define como "la co-construcción, entendiendo por tal una labor conjunta entre los implicados en el problema/solución (según el caso, interequipo, entre profesionales de un mismo servicio, o entre servicios, o en toda la red) donde los actores asumen alternativamente roles iguales y diferentes".

- Las normas y los valores deben ser compartidos en el proceso de intervención: para Ferreira (2011: 135), el modelo de intervención en redes "implica un cambio en la toma de decisiones, en la medida en que implica una horizontalidad en la acción y en la toma de decisiones"; y para Llobet (2004: 74), aprender a trabajar en red y de forma interdisciplinaria supone un ejercicio de reconocimiento de las capacidades de los demás, de poner en práctica la capacidad de escucha y de dialogo, de trabajar los liderazgos compartidos, al permitir "trabajar la motivación desde una lógica de sistema, es decir, de retroalimentación y/o apoyo de la motivación, especialmente en los momentos críticos, duros o difíciles que se puedan dar”. Ahora bien, es necesario preguntarnos qué supone la interdisplinariedad: "la interdisciplinariedad supone una ética de confianza, basada en el principio de la relación humana y en el compartir los valores y las normas que rigen la intervención profesional" (Ferreira, 2011: 228).

- Permite dar respuestas integrales a la realidad de nuestro tiempo: la intervención profesional debe plantearse como objetivo ayudar al individuo, al grupo y a la comunidad a utilizar "los recursos necesarios para resolver su propio estado de necesidad; recursos que son, en primer lugar, personales (la propia capacidad de reaccionar y de afrontar los problemas), pero también ambientales (la capacidad de entrar en contacto y utilizar los sistemas de ayuda natural, las redes de apoyo social) y sociales (los recursos institucionales organizados en servicios, programas y prestaciones)" (Del Pino, 2000: 285). Los profesionales trabajan en organizaciones que tienen ciertas estructuras de funcionamiento, 
normalmente poco permeables a los cambios y donde, según afirma Kanter (1994), la colaboración entre diferentes organizaciones es compleja, puede darse de diferentes formas, pero siempre es difícil de construir. Como señala Kern (2003: 58), "en lo vivencial y en la construcción de relaciones, que tienen como base lo simbólico y lo imaginario, el poder institucional constituye un elemento básico para la comprensión de las relaciones interpersonales". Una organización es un fenómeno indescriptible si se pretende una descripción exhaustiva y objetiva; una organización “es, en definitiva, la descripción de un observador, una metáfora, por tanto, [...] contiene reflexivamente el contexto psicosocial del mismo" (Castillo, 1997: 224). La interdisciplinariedad "implica una mayor riqueza respecto a los resultados del trabajo, aunque supone un mayor esfuerzo, al poner sobre la mesa distintos lenguajes, modos de hacer y puntos de vista" (Bautista, 2011: 178).

- Fomenta la articulación entre las instituciones públicas y privadas: articular redes entre sí, formales o informales, para aumentar la participación, la fuerza y la información colectiva, "crea condiciones para cambiar la visión de los problemas presentes y las condiciones particulares de su producción" (Faleiros, 2003: 105). Sin embargo, lo nuevo no es la existencia de redes (Aguilar, 2005), sino las posibilidades de transformación de las redes sociales individuales en redes de estructura social. Se trata de un cambio cuantitativo, pero también cualitativo, ya que cambia la estructura de la sociedad y, a su vez, las formas de exclusión social. Si la estructura social es un sistema multidimensional, en redes, que opera en un sistema digital, la mayor exclusión social es la exclusión de las redes. La organización de la intervención profesional en red "posibilita innovar/crear lo contrario a una filosofía de trabajo aislado; fomenta la articulación entre las organizaciones (públicas/privadas) y los diferentes grupos, intentando encontrar respuestas adecuadas a las necesidades derivadas de las cuestiones sociales generadas por la estructura socioeconómica” (Ferreira, 2011: 284). Sin embargo, "lo cierto es que se trata aún de una perspectiva más ligada a lo que creemos que debería ser que a lo que en realidad es" (Llena, Parcerisa, y Úcar, 2009: 92).

El presente artículo presenta una reflexión sobre la necesidad de realizar un trabajo transversal y en red por parte de los trabajadores sociales y demás profesionales que intervienen en el ámbito local desde los servicios sociales comunitarios, incorporando a dicho análisis los resultados de entrevistas semiestructuradas a profesionales, y pretende avanzar en la definición del trabajo interdisciplinar y conocer los niveles de intervención en los que desarrollan su trabajo y consideran que tendrían que desarrollarlo, según su visión en el momento actual.

\section{Método}

La base de este artículo es una investigación empírica realizada mediante entrevistas semiestructuradas a treinta trabajadores sociales con más de diez años de experiencia en el ámbito local; cinco técnicos responsables del área de Bienestar Social municipal; cinco profesionales de otras disciplinas que realizan programas transversales con trabajadores sociales y cinco representantes de organizaciones no lucrativas que desarrollan su actividad en el ámbito local. El estudio fue realizado en las localidades de más de cien mil habitantes de la Comunidad de Madrid (Alcalá de Henares, Alcobendas, Alcorcón, Fuenlabrada, Getafe, Leganés, Móstoles, Parla y Torrejón de Ardoz). En el curso de las entrevistas semiestructuradas, se pidió a estos profesionales que definieran el trabajo interdisciplinar, el nivel o niveles de intervención en los que consideraban que se trabaja en el ámbito local y los niveles en los debería realizarse la intervención desde el ámbito municipal. Estos datos se obtuvieron mediante tres preguntas: una primera que pedía a los profesionales que clasificaran una serie de afirmaciones según un baremo de importancia (1, 'nada importante'; 2, 'poco importante'; 3, 'bastante importante'; y 4, 'muy importante'; o bien, ninguna de las opciones señaladas); una segunda donde se pedía la valoración de todos los actores encuestados en relación al nivel o niveles de intervención en los que consideraban que se trabaja en el ámbito local (individual, colectivo, en coordinación y/o en red); y una tercera pregunta sobre los niveles en los que consideraban que debería realizarse su intervención desde el ámbito municipal

Con respecto a la elección de la muestra, buscábamos que la información obtenida fuese extrapolable a la totalidad de los trabajadores sociales que prestan sus servicios en la Administración Local y consideramos relevante la experiencia laboral de los profesionales en el ámbito de la atención primaria, pues “hace que las opiniones recibidas se sustenten en un amplio conocimiento por parte de los profesionales, así como su capacidad para detectar debilidades, amenazas, fortalezas y oportunidades del sistema" (Subirats, 2007: 60); igualmente, se pretendía recoger la especificidad de municipios diferentes, porque los servicios desarrollados con un enfoque comunitario permiten reconocer la diversidad de las personas y del territorio en el que se producen las desigualdades y emergen nuevos riesgos de vulnerabilidad. Atendiendo a la máxima representatividad de los discursos existentes, se consideró necesario un análisis que contemplara la diversidad de los diferentes actores implicados, es decir, actores técnicos, profesionales y asociativos.

Para el análisis cuantitativo de las respuestas, se utilizó el programa informático Statistical Package for Social Sciences (SPSS 20), que nos sirvió de soporte para la codificación y análisis de resultados. La posibilidad de organizar la información proveniente de diferentes actores (trabajadores sociales, técnicos, profesionales y responsables de asociaciones) nos permitió avanzar en la comprensión de los resultados. 


\section{Resultados}

Hemos preguntado a los trabajadores sociales y demás actores encuestados por la definición del trabajo interdisciplinar que realizan en el ámbito local. El Gráfico 1 nos muestra que la definición preferida para el $70 \%$ de los trabajadores sociales es "un proceso de co-construcción (labor conjunta de todos los implicados en el problema/solución)", definición valorada de forma unánime por los demás actores encuestados. El 63,3\% de los trabajadores sociales han considerado también que "permite dar respuestas integrales a la realidad de nuestro tiempo". Nos parece claro señalar que "los servicios sociales comunitarios han de ser observados compartiendo cotidianidad al lado de otras instituciones públicas (colegios, centros de salud, policía local), de entidades vecinales, sociales" (Jaraíz, 2012: 121), y que "el 'municipio relacional' debe abrir las puertas a una pluralidad de actores y facilitar la incorporación en las tareas de gestión y prestación de los servicios locales" (Izquieta, 2011: 53).

Los técnicos, a su vez, han valorado especialmente la definición de "normas y los valores compartidos en el proceso de intervención" y los profesionales, al igual que los representantes de organizaciones no lucrativas, consideran que "permite dar respuestas integrales a la realidad de nuestro tiempo". Los diferentes actores han coincidido de nuevo en las dos opciones menos valoradas: "necesidad de compartir conocimientos y liderazgo" y "fomentar la articulación entre las instituciones públicas y privadas”. En el contexto social y cultural en el que el ser humano construye sus relaciones desde la perspectiva de las redes sociales, se expresa "un mundo relacional que sólo se puede percibir y justificar desde la necesidad intrínseca que tiene todo ser humano de vivenciar y experimentar el formar parte e insertarse en una red que responda a sus necesidades" (Kern, 2003: 50-51); y, en el momento presente, "estar en red, no es una opción, sino un requisito de constitución del propio servicio, ya que el tratamiento (entendido como el conjunto de acciones que abordan una situación definida como problemática) es la red misma" (Ubieto, 2009: 40).

La era de la globalización trae consigo un nuevo orden social que influye en la actuación de los trabajadores sociales, dado que hoy "la acción social está basada en proyectos y presupuestos, en la responsabilidad individual y social del ciudadano, en un nuevo modelo organizacional (descentralización) y también en un nuevo modelo de intervención (sociedad/ redes)" (Ferreira, 2008: 39), y las redes sociales pueden ser el motor de la participación constructiva en los procesos comunitarios locales y constituyen un verdadero potencial en la promoción de la participación y en la autonomía de la persona.

La actividad del trabajador social debe tener como base el valor de la persona humana y su potencial de desarrollo y cambio en los cuatro niveles de intervención, que se complementan, y a partir de los cuales los trabajadores sociales pueden realizar su actividad en el ámbito local: individual, colectivo, en coordinación y en red. Así pues, hemos preguntado a todos los actores cuál es el nivel o los niveles de intervención en los que consideran que se trabaja en el ámbito local (Gráfico 2) y cuáles son los niveles en los que consideran que debería realizarse su intervención desde el ámbito municipal (Gráfico 3).

Los trabajadores sociales han considerado como niveles menos frecuentes de intervención el colectivo y en red, pero por el contrario, a la pregunta relativa a los niveles más adecuados para trabajar desde el ámbito local (Gráfico 3) han respondido que debería

Gráfico 1. Definición de trabajo interdisciplinar por parte de los actores entrevistados (\%)

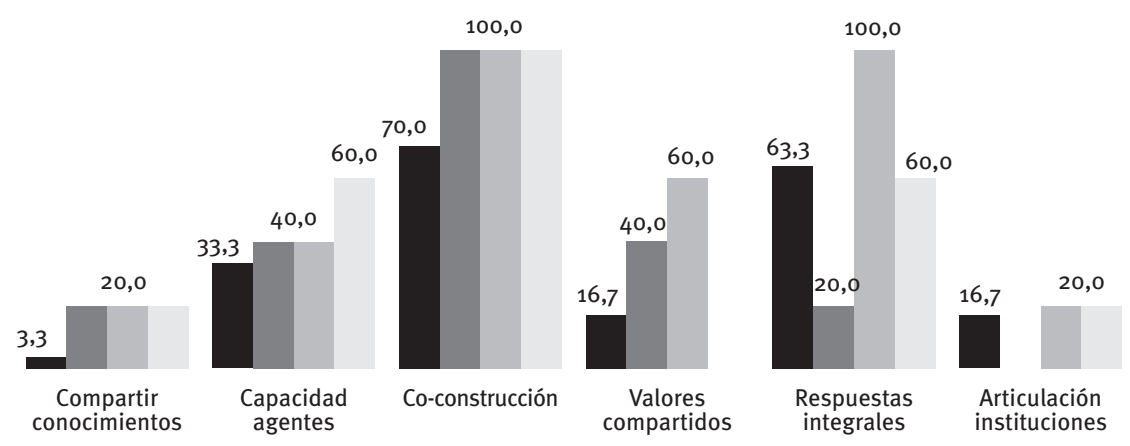

Trabajadores sociales

Técnicos responsables de Bienestar Social

Profesionales de otras disciplinas

Representantes de organizaciones no lucrativas

Fuente: Rodríguez Álvarez (2014). 


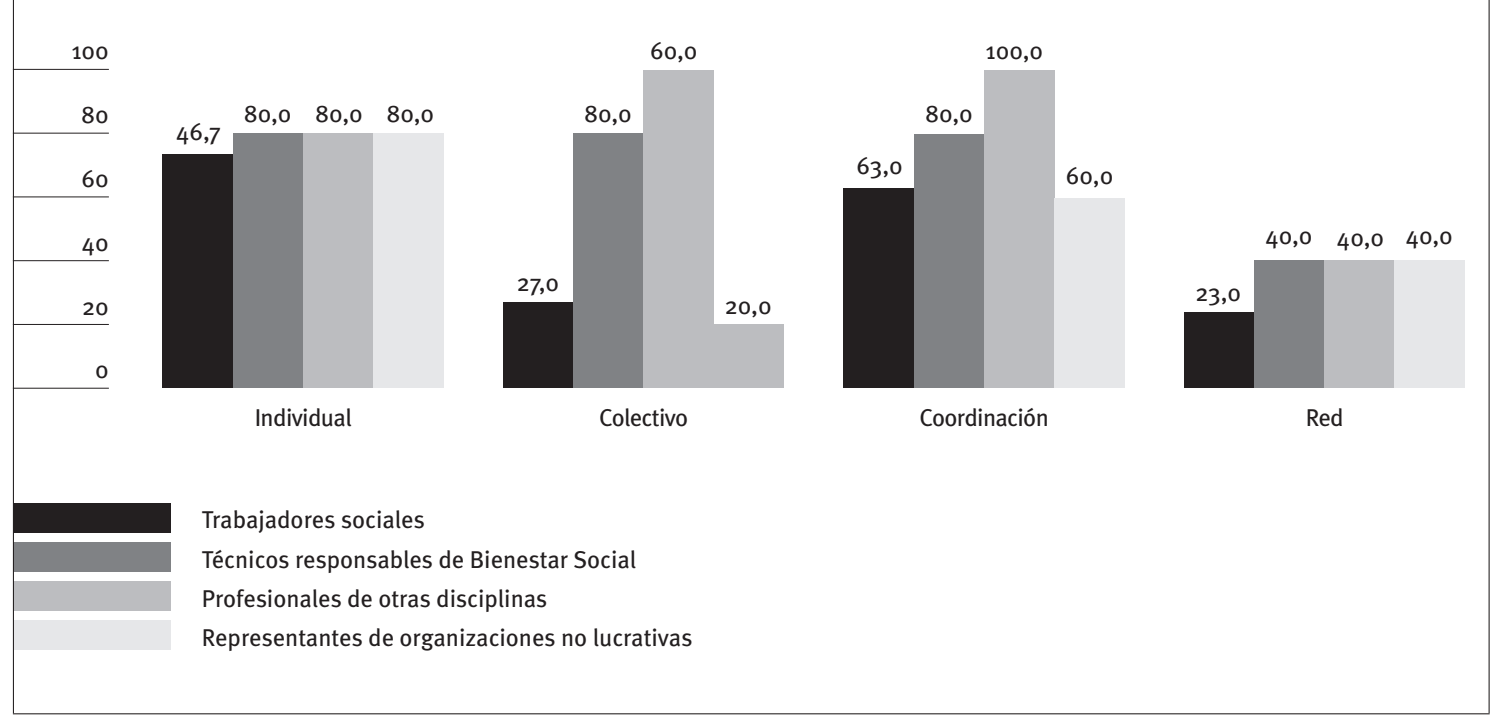

Fuente: Rodríguez Álvarez (2014).

Gráfico 3. Niveles de intervención en los que se debería trabajar en el ámbito local (\%)

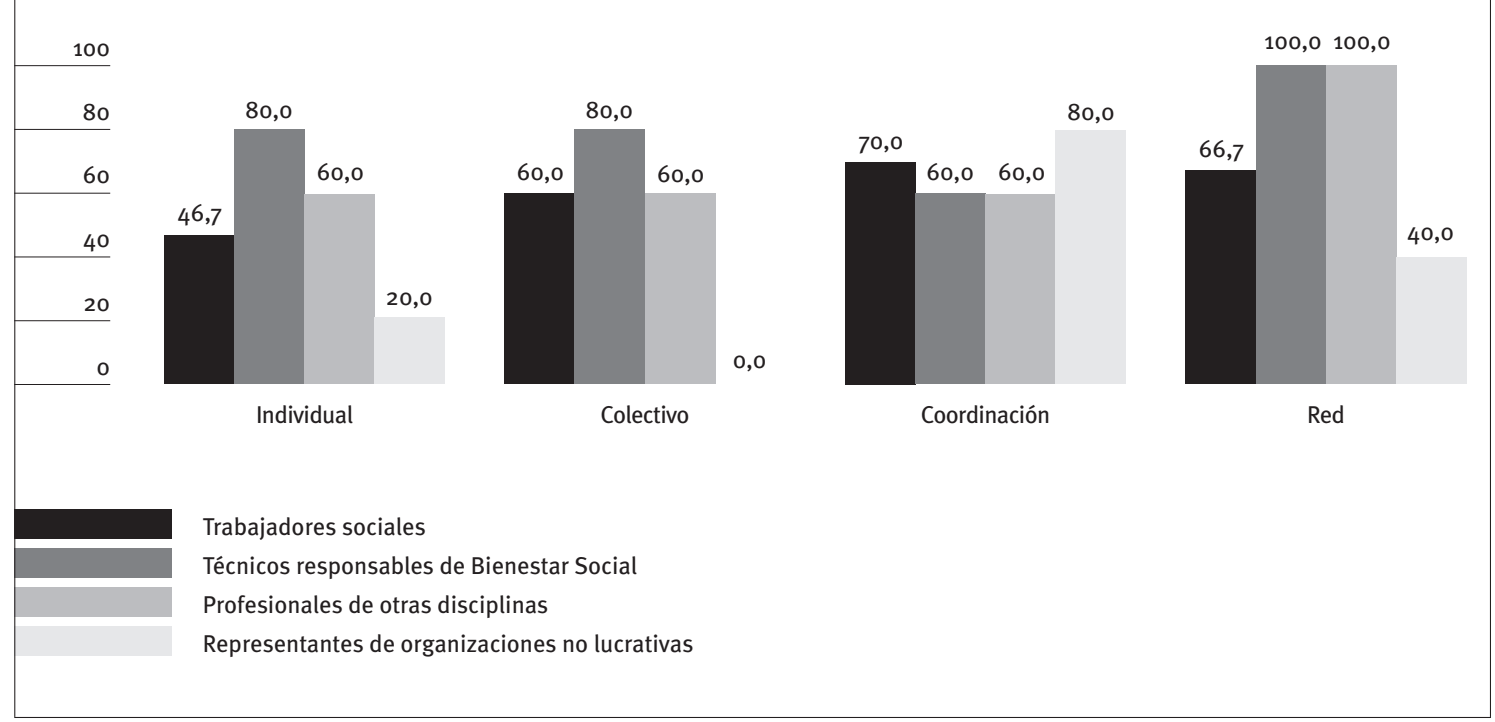

Fuente: Rodríguez Álvarez (2014).

ser en red prioritariamente, en coordinación con otras instituciones, a nivel colectivo y, en último lugar, a nivel individual. Un aspecto que consideramos decisivo para el trabajo social comunitario en el ámbito local es poner en el centro de la acción social a la persona, "porque para que exista un desarrollo verdadero, la persona debe permanecer en una posición activa, y esta posición debe ser impulsada por el trabajador social, a partir de la consideración del sujeto como ciudadano, al tener en cuenta sus aspectos individuales y su dimensión social y colectiva, y no únicamente como usuario, beneficiario o cliente (Rodríguez Álvarez, 2015: 194). Los técnicos y los profesionales han coincidido en subrayar que se trabaja poco en red, y todos los encuestados han señalado que debería ser el nivel prioritario de intervención. Los representantes de organizaciones no lucrativas, a su vez, han considerado mayoritariamente que se trabaja a nivel individual y en coordinación, y que debería trabajarse en coordinación con otras instituciones; un $40 \%$ consideran que debería trabajarse en red; y ninguno ha señalado el trabajo colectivo como importante.

Sin embargo, la praxis actual del trabajo social revela un claro predominio del trabajo individual-familiar frente al comunitario: "el modelo de actuación debe abarcar los tres ámbitos y basarse en contextos de colaboración. Pese a esta aseveración, la percepción es que el trabajo individual es el que ocupa 
la mayor parte de la jornada y no deja tiempo para la planificación y la prevención" (Subirats, 2007: 57-58). No obstante, es importante señalar que "es la intervención comunitaria la que se caracteriza, entre otros aspectos, por la incorporación de los recursos naturales de las personas, familias y grupos de una comunidad en los procesos de resolución y prevención de los problemas sociales" (Rodríguez Álvarez, 2015: 193), porque "no podemos afrontar los cambios solos y tampoco en grupos pequeños: los retos estructurales demandan respuestas estructurales, y precisamente en ese ámbito recupera toda su vigencia el trabajo social comunitario" (López Peláez y Segado, 2012: 234).

Podemos considerar la metodología del trabajo en red una manera efectiva de compartir información, de aprender de la experiencia del otro, de trabajar juntos facilitando las iniciativas de los miembros y creando un efecto multiplicador en el que la diversidad es una riqueza. Para Rodrigo et al. (2008), las redes implican: a) el aprendizaje cooperativo entre sus miembros (aceptación de la diferencia, trabajo en equipo, articulación, colaboración); b) la adaptación a las realidades locales (conocimiento de las necesidades y demandas, comprensión de las dinámicas locales); y c) el trabajo efectivo (metodología, mantenimiento de la motivación y capacidad de plantearse proyectos realistas y viables). En suma, "las redes pueden ser el motor de la participación constructiva en los procesos comunitarios locales" (ibídem: 142).

\section{Conclusiones}

A la largo del presente artículo hemos analizado la intervención del trabajador social, y de otros profesionales, en el ámbito de los servicios sociales comunitarios, subrayando la importancia de algunas afirmaciones relativas al trabajo interdisciplinar y a los diferentes niveles de intervención que realizan los profesionales -en concreto, el nivel individual, el colectivo, el trabajo en coordinación y el trabajo en red-para cumplir su objetivo de activar los recursos de las personas y de los diferentes contextos en los que intervienen. Los profesionales encuestados han coincidido en considerar de manera especial que el trabajo interdisciplinar es "un proceso de co-construcción (labor conjunta de todos los implicados en el problema/solución)" y, a su vez, que el nivel más adecuado para trabajar desde el ámbito local debería ser en red prioritariamente, excepto los representantes de organizaciones no lucrativas, que han considerado que debería trabajarse, en primer lugar, en coordinación con otras instituciones.

Entendemos que es necesario potenciar el trabajo de redes sociales desde una perspectiva global, estimulando la conexión de las redes primarias con otros escenarios y actores, para que, a través de la interacción y el establecimiento de lazos internos y externos, de solidaridad y colaboración, enfrenten las situaciones amenazantes, sin olvidar la necesidad que tienen los trabajadores sociales de contar con “un conocimiento teórico-práctico y capacidad de intervención que garantice el equilibrio entre las tres principales dimensiones de la intervención desde el territorio: planificación y evaluación, cooperación institucional e intervención próxima a los individuos y a las comunidades" (Cardoso, 2012: 210).

A la luz de los resultados obtenidos, podemos concluir afirmando, a modo de síntesis, la necesidad de contar en el ámbito de los servicios sociales con una metodología de intervención interdisciplinar realizada en 'co-construcción', una ética de la confianza basada en el principio de la relación humana y en compartir los valores que rigen la intervención profesional, metodología que implica un enriquecimiento de los resultados del trabajo y debe realizarse desde una cultura organizativa no pensada para funcionar en red, pero que necesita dar una respuesta integral a problemas sociales multidimensionales y requiere una nueva articulación de instrumental práctico. Sin duda alguna, la interdisciplinariedad "garantiza que cada profesional, en su intervención concreta, es capaz de considerar la complejidad de lo social" (Acebo, 1992: 102). 


\section{Bibliografía referenciada}

ACEBO, Á. (1992): Trabajo social en los servicios sociales comunitarios. Análisis del momento actual y búsqueda de posibilidades de mejora desde la óptica de un servicio social de base, Madrid, Consejo General de Colegios Oficiales de Diplomados en Trabajo Social y Asistentes Sociales; Siglo XXI de España.

AGUILAR, M. J. (2005): “Dinamización, animación sociocultural y desarrollo comunitario: convergencias, divergencias y retos de futuro", en ÚCAR, X. (ed.), Participación, animación e intervención socioeducativa, Barcelona, Universitat Autónoma, págs. 1-22.

ALEMÁN, C. (2010): "Situación actual y perspectivas de los servicios sociales en España”, en ALEMÁN C. (coord.), Fundamentos de Servicios Sociales, Valencia, Tirant lo Blanch, págs. 299-308.

BAUTISTA, M. J. (2011): “Competencias y profesionalización. Interdisciplinariedad y trabajo en equipo", en PÉREZ SERRANO, G. (coord.), Intervención Sociocomunitaria, Madrid, Universidad Nacional de Educación a Distancia, págs. 165-182.

CARDOSO, J. F. (2012): “Acção Social nos Municípios portugueses, potencialidades e limitações" [tesis doctoral], Lisboa, Instituto Universitário de Lisboa, Departamento de Ciência Política e Políticas Públicas.

CASADO, D. (2007): "Perfeccionamiento de los Servicios sociales en España: informe con ocasión de la ley sobre autonomía y dependencia", en CASADO, D. y FANTOVA, F. (coords.), Perfeccionamiento de los servicios sociales en España: informe con ocasión de la Ley sobre Autonomía y Dependencia, Madrid, Cáritas Española, págs. 255-288.
CASTILLO, F. (1997): “El profesional en las organizaciones de servicios sociales", en COLETTI M.; y LINARES, J. L. (comps.), La intervención sistémica en los servicios sociales ante la familia multiproblemática, Barcelona, Paidós, págs. 224-243.

COMUNIDAD DE MADRID (2003): “Ley 11/2003, de 27 de marzo, de Servicios Sociales de la Comunidad de Madrid”, Boletín Oficial de la Comunidad de Madrid, no- 88, 14-4-2003, págs. 6-22.

DE LA RED, N. (1997): “Política Social y Trabajo Social”, en ALEMÁN, C.; y GARCÉS, J. (coords.), Política Social, Madrid, McGraw-Hill, págs. 531-552.

DE ROBERTIS, C. (2006): Metodología de intervención en Trabajo Social, Buenos Aires, Lumen Humanitas.

DEL PINO, M. D. (2000): “La dimensión individual y colectiva del trabajo social en los servicios sociales comunitarios", en MARTíNEZ, $M^{\underline{a}}$ J. (coord.), Para el trabajo social: aportaciones teóricas y prácticas, Granada, Maristán, págs. 275-300.

FALEIROS, V. de P. (2003): Estrategias de empowerment en Trabajo Social, Buenos Aires, Lumen Humanitas.

FERRARIO, F. (2009): Il lavoro di rete nel servizio sociale. Gli operatori fra solidarietà e istituzioni, 5ํㅡㄹ. ed., Roma, Carocci.

FERREIRA, J. M. L. (2011): Serviço social e Modelos de Bemestar para a Infância. 'Modus operandi' do Assistente Social na Promoçao da Protecção à Criança e à Familia, Lisboa, Ouid Juris.

- (2009): "Pensar a formação em Serviço Social no quadro da globalização e do espaço único europeu", Intervenção Social, nํㅜ 35, págs. 351-366. 
- (2008): "Trajetórias e produção do conhecimento do Serviço Social Português. 0 papel do Instituto Superior de Serviço Social de Lisboa - ISSSL", Intervenção Social, no 32-34, págs. 35-54.

GUTIÉRREZ RESA, A.; y UÑA, O. (2010): Integración de los inmigrantes a través de los servicios sociales municipales en la Comunidad de Madrid, Madrid, Universidad Nacional de Educación a Distancia.

IZQUIETA, J. L. (2011): Voluntariado y Tercer Sector. Cultura, participación cívica y organizaciones solidarias, Madrid, Tecnos.

JARAÍZ, G. (2012): Intervención social, barrio y servicios sociales comunitarios, Madrid, Fundación FOESSA, Cáritas.

KANTER, R. M. (1994): “Collaborative advantage: The art of alliances", Harvard Business Review, vol. 72, no ${ }^{4}$, págs. 96-108.

KERN, F. A. (2003): As mediações em redes como estratégia metodológica do serviço social, Porto Alegre, Edipurs.

LLENA, A.; PARCERISA, A.; y ÚCAR, X. (2009): 10 ideas clave. La acción comunitaria, Barcelona, Graó.

LLOBET, M. (2004): “El Trabajo Social comunitario como una oportunidad y estrategia para poder re-pensar, trans-formar y co-construir la organización de la atención primaria en Servicios Sociales", Revista de Servicios Sociales y Política Social, nํ6 66, págs. 63-76.

LÓPEZ PELÁEZ, A.; y SEGADO, S. (2012): “Personas, grupos, sociedades: perspectivas para la intervención social comunitaria en el siglo XXI", en FOMBUENA, J. (coord.), El trabajo social y sus instrumentos: elementos para una interpretación 'a piacere', Valencia, Nau Llibres, págs. 227-248.

NAVARRO, S. (2004): Redes sociales y construcción comunitaria, Madrid, CCS.

RODRIGO, M. J. et al. (2008): Preservación familiar. Un enfoque positivo para la intervención con familias, Madrid, Pirámide.

RODRÍGUEZ ÁLVAREZ, M. D. (2015): “El trabajo social comunitario en el ámbito local: protagonismo colectivo y nuevas realidades de convivencia", Documentación Social, no 173, págs. 189-204.

- (2014): "Redes sociales: un nuevo cuadro metodológico de intervención en Trabajo Social Comunitario" [tesis doctoral], Madrid, Universidad Complutense de Madrid, Departamento de Trabajo Social y Servicios Sociales.

RODRÍGUEZ CABRERO, G. (dir.) (2011): Servicios Sociales y cohesión social, Madrid, Consejo Económico y Social.

RYGAARD, N. P. (2008). El niño abandonado. Guía para el tratamiento de los trastornos del apego, Barcelona, Gedisa.

SUBIRATS, J. (dir.) (2007): Los Servicios Sociales de Atención Primaria ante el cambio social, Madrid, Ministerio de Trabajo y Seguridad Social.

UBIETO, J. R. (2009): El trabajo en red. Usos posibles en Educación, Salud Mental y Servicios Sociales, Barcelona, Gedisa.

VEGA, S. (1997): "Instrumentos de trabajo", en COLETTI, M.; y LINARES, J. L. (comps.), La intervención sistémica en los servicios sociales ante la familia multiproblemática, Barcelona, Paidós, págs. 167-200. 